Erschienen in: Hoffmann, Ludger (Hrsg.): Deutsche Syntax. Ansichten und Aussichten. - Berlin, Boston: de Gruyter, 1992. S. 435-457. (Institut für deutsche Sprache. Jahrbuch 1991)

INGER ROSENGREN

\title{
Satztyp, Satzmodus und Illokution aus modularer Sicht
}

\section{Das Problem}

In der traditionellen Grammatik (siehe u.a. Duden (1984), Helbig/Buscha (1991), Grundzüge (1981)) wird zwischen mindestens fünf Satztypen oder Satzarten unterschieden: Aussagesatz, Fragesatz, Aufforderungssatz, Ausrufesatz (oder wie man ihn auch nennt Exklamativsatz) und Wunschsatz. Wie die Termini zeigen, handelt es sich dabei um eine funktionale und formale Klassifizierung von Sätzen zugleich, wobei die Funktion terminologisch die Hauptrolle zu spielen scheint. In der Forschung der letzten Jahre wird ein Versuch gemacht, diese beiden Aspekte oder Beschreibungsebenen mit Hilfe eines neuen Begriffs, des Begriffs des Satzmodus (zuweilen anders benannt), zu trennen (vgl. hierzu vor allem die Beiträge in Meibauer (1987) und in Studien zum Satzmodus I, II und III (1988; 1989); siehe auch Altmann (1990), Altmann/Batliner/Oppenrieder (1989), Brandt/Rosengren/Zimmermann (1989), Grewendorf/Zaefferer (demnächst), Motsch/Reis/Rosengren (1989), Motsch/Pasch (1987), Pasch $(1989 ; 1990 a, b)$. Der Satzmodus ist die semantische Vermittlungsinstanz zwischen den formalen Eigenschaften des Satztyps und seinem Sprechaktpotential. Über die Richtigkeit einer solchen grundlegenden modularen Herangehensweise ist man sich heute wohl prinzipiell einig. Dissens besteht jedoch in Bezug auf die Beantwortung folgender Fragen:

(a) Welche Eigenschaften von Sätzen sind als Satztypmerkmale zu betrachten?

(b) Wie viele und welche Satztypen gibt es?

(c) Was genau ist unter dem Begriff des Satzmodus zu verstehen?

(d) Haben alle Sätze einen Satzmodus?

(e) Wie kommt man vom Satzmodus zur Mlokution?

Die Beantwortung von (a) determiniert die Beantwortung von (b). Bei (c) geht es darum, ob der Satzmodus eine Sprechereinstellung oder möglicherweise ein einstellungsfreier Operator ist, und von der Beantwortung dieser Frage hängt wiederum ab (d), ob z.B. Nebensätze auch einen Satzmodus haben. Die Beantwortung der letzten Frage (e) ist besonders schwierig, weil wir mit ihr den vergleichsweise einigermaßen gut beschriebenen Bereich der Grammatik verlassen und uns in das unsichere und noch wenig erforschte Feld der Pragmatik hineinbegeben. 
Ich werde mich im folgenden diesen fünf Fragen widmen - wobei ich auf die gemeinsamen Resultate des Programms "Sprache und Pragmatik" zurückgreife (siehe hierzu die im Literaturverzeichnis aufgeführten Arbeitsberichte des Programms) ${ }^{1}$ - und werde dafür argumentieren,

(a) daß es nur drei grundlegende Satztypen gibt,

(b) daf jeder Satz einen Satzmodus hat,

(c) daß der Satzmodus einstellungsfrei ist, und

(d) daß - folglich - die Einstellung erst auf der illokutiven Ebene hinzukommt.

\section{Das Grammatik:Pragmatik-Verhältnis}

Ehe ich mich dieser Aufgabe zuwende, will ich ein paar allgemeine Überlegungen zu dem Verhältnis zwischen Grammatik und Pragmatik vorausschicken: Ich lege die Annahme zugrunde, daß das Verhältnis zwischen Grammatik und Pragmatik modular ist (siehe hierzu ausführlicher Motsch/Reis/Rosengren (1989) und dort angeführte Literatur). Sie stehen in einem autonomen und zugleich interdependenten Verhältnis zueinander. Mit autonom soll gemeint sein, daß die beiden Module (möglicherweise Systeme von Modulen) eigenständige Kenntnissysteme sind, d.h. gekennzeichnet sind durch je eigene Prinzipien, Einheiten und Regeln, die sich nicht auf die Prinzipien, Einheiten und Regeln des jeweiligen anderen Moduls reduzieren lassen. Mit interdependent soll gemeint sein, daß die pragmatischen Funktionen durch die Outputeinheiten der Grammatik realisiert werden, diese Outputeinheiten ihrerseits aber nur als pragmatische Einheiten aktualisiert werden können. Es gibt also keine Pragmatik ohne Grammatik. Andererseits ist die Grammatik eine Service-Instanz für die kommunikativen Aufgaben, die in der Pragmatik definiert werden.

Während die Grammatik sicherlich die Repräsentationsebenen oder Komponenten Syntax, Semantik, Phonologie und Lexikon umfaßt, die ihrerseits in einem systematischen Verhältnis zueinander stehen, ist die Gliederung der Pragmatik noch weitgehend ungeklärt. Hier soll jedoch davon ausgegangen werden, daß die Pragmatik zumindest über ein Illokutionssystem (-modul) verfügt, das in einem systematischen Verhältnis zu dem Satz als Output der Grammatik steht.

1 Nach Abschluß des vorliegenden Beitrags ist noch ein größerer Aufsatz im Rahmen des Programms entstanden: Brandt/Reis/Rosengren/Zimmermann (1992). In diesem Aufsatz werden die hier vorgetragenen Grundgedanken weiter entwickelt und theoretisch untermauert. $\mathrm{Zu}$ Einzelproblemen siebe Fries (1992), Rehbock (1992a,b), Reis (1992), Rosengren (1992a). 
Auf diesem Hintergrund ist der Satz ein lexikalisch spezifiziertes Gebilde mit einer syntaktischen, semantischen und phonologischen Struktur, das losgelöst von der Äußerungssituation nur eine grammatisch determinierte Bedeutung hat. Diese legt ein kommunikatives Potential fest, aus dem im Augenblick der Äußerung eine der möglichen Anwendungen herausgegriffen wird, wobei der Satz auf einen Sachverhalt bezogen wird und eine Ẩerungsbedeutung erhält (siehe Bierwisch 1979; 1980). Darüber hinaus wird mit der Äußerung ein Interaktionsziel verfolgt (ein illokutiver Akt vollzogen).

\section{Zum Begrifi des Satztyps}

3.1. Bisher wurde nur ganz allgemein vom Satz und seinem kommunikativen Potential gesprochen. Die in diesem Zusammenhang sich stellende Frage ist nun, welche phonologischen, syntaktischen und semantischen Eigenschaften des Satzes für seine illokutive Funktion zuständig sind. In diesem Abschnitt soll vorerst untersucht werden, ob es überhaupt möglich ist, Satztypen konsistent zu definieren, und gegebenenfalls, welche Kriterien für eine solche Definition relevant sind. Auf der Suche nach Kriterien fallen uns Stellung des Verbs, der Verbmodus und die Füllung des eventuell existierenden Vorfelds mit einer XP-Phrase ( \pm w-Phrase) ein. Folgende Fragen stellen sich:

(a) Können wir mit diesen an der Oberfläche sichtbaren Eigenschaften von Sätzen einzelne Satztypen unterscheiden?

(b) Ist die eventuell mögliche Klassifizierung intuitiv überzeugend, bzw. expliziert sie die in der traditionellen Grammatik unterstellte Beziehung zwischen Form und Funktion?

Unsere traditionelle grammatische Schulung beeinfluBt sicherlich die Erwartungen, die wir bezüglich einer Satztypologie haben. Vielleicht spiegelt die Tradition aber auch die Sprachkompetenz. Wie dem auch sei: Die folgenden Beispiele (Verb-erst-, Verb-2weit- und selbständig gebrauchte Verb-letzt-Sätze) geben uns nicht viel Hoffnung, daß wir mit oberflächenstrukturellen Kriterien eine intuitiv überzeugende Satztypologie erstellen können:

(1) Ein Mann kam zur Tür herein.

(2) Kam ein Mann zur Tür herein./?

(3) Kommt der morgen?

(4) Der kommt morgen?

(5) Der zieht sich (doch) komisch an.

(6) Der zieht sich (aber/vielleicht) komisch an!

(7) Zieht der sich (aber/vielleicht/*doch) komisch an! 
(8) Daß du ja dort wegbleibst!

(9) Daß der dort wegbleibt!

(10) Wen der alles kennt!

(11) Wen kennt der alles (nicht)?

(12) Wen kennt der nicht alles!

(13) Wen der nicht alles kennt!

(14) Komm doch her!

(15) Kommen Sie doch her!

(16) Das Buch lassen Sie bitte liegen!

Die Beispiele zeigen, daß Oberflächenkriterien wie Verbstellung, Verbmodus, Vorfeld mit XP-Phrase ( \pm w-Phrase) eine merkwürdige Klassifizierung ergeben, die zumindest nicht mit der der traditionellen Grammatik übereinstimmt. So gibt es Deklarativsätze mit Verb-erst, die sich oberflächenstrukturell nicht von Entscheidungsinterrogativsätzen (E-IS) und den sogenannnten Exklamativsätzen unterscheiden lassen ((2)-(3), (7)). Der w-IS (11) scheint durch die satzinitiale w-Phrase enger mit den durch w-Phrasen eingeleiteten Exklamativsätzen (10), (12)-(13) zusammenzugehören als mit dem E-IS (3). Die Exklamativsätze (6)-(7), (9)(10), (12)-(13) ihrerseits bilden gar keine eigene formale Klasse. Jeder Strukturtyp kommt auch als Exklamativsatz vor. Zwar hat der Imperativsatz - zumindest im prototypischen Fall (14) - eine eigene Verbform, was öfter dazu geführt hat, daß man die Satztypen mit Hilfe des Merkmals timp in zwei Gruppen unterteilt (siehe u.a. Rosengren (1988a)). Eine solche Untergliederung der Satztypen bedeutet aber, daß man den Deklarativsatz und den Interrogativsatz negativ als nicht-imperativisch definiert, was merkwürdig anmutet.

Wenn wir vorläufig vom sogenannten Exklamativsatz absehen (siehe hierzu Rosengren (1992a)), kann man auch nicht durch Hinzunahme des Tonmusters eine eindeutige und überzeugende Klassifizierung erhalten. Zwar ist der steigende Tonverlauf prototypisch für den E-IS. Nicht jeder E-IS wird aber mit steigender Intonation gesprochen. Der w-IS weist seinerseits auch nicht immer fallende Intonation auf. Unklar ist übrigens auch die Einordnung des Assertionsfragesatzes (4), der steigende Intonation verlangt. Die Intonation hilft uns gewiß bei der Disambiguierung der Sätze nach Satztypen, sie ist aber als Definitionsmerkmal weder notwendig noch hinreichend.

Auch die Distribution der Modalpartikeln ist kein zuverlässiges Kriterium, weil es sich nur um eine hohe, aber keine durchgängige Korrelation zwischen Satztyp und Modalpartikelselektion handelt und die Modalpartikeln darüber hinaus nur selten obligatorisch sind. 
Irgendwie scheinen wir also nicht auf dem richtigen Weg zu sein, wenn wir versuchen, die Satztypen mit Hilfe von oberfächenstrukturellen Eigenschaften wie Verbstellung, Besetzung des Vorfelds und Intonation zu definieren. DaB eine nach diesen Kriterien vorgenommene Klassifizierung nicht mit der traditionellen Einteilung übereinstimmt, wäre an sich nicht ausschlaggebend. Sie ist aber darüber hinaus inkonsistent und ad hoc.

In dieser Situation bleiben eigentlich nur zwei Möglichkeiten:

(a) Es gibt gar keine syntaktischen Satztypen im traditionellen Sinn, sondern nur Satzmodi, die mit bestimmten Oberfächenmerkmalkonfigurationen korrelieren.

(b) Die Sätze weisen auf einer tieferen Ebene syntaktische Merkmale auf, die die Oberfächenstrukturen determinieren und die Träger der Satzmodi sind.

Für jede dieser Thesen gibt es Vertreter. Welche Konsequenzen die erste These hat, wird erst richtig deutlich, wenn man versucht, sie theoretisch auszubuchstabieren und empirisch zu prüfen. Ein solches ausbuchstabiertes Modell hat für den Bereich des w-IS Pasch (1991) entworfen. Wie Reis (1991b) zeigt, läßt sich jedoch eine Reihe von Einwänden gegen eine solche Lösung vorbringen, die zusammengenommen zeigen, daß dieser Weg vermutlich nicht zum Ziel führen kann. Bleibt noch der zweite Weg, den ich im folgenden gehen werde.

3.2. Ich werde also zu zeigen versuchen, daß die These (b) sich erhärten läßt, wobei sich auch herausstellen wird, daß die traditionelle Klassifizierung nicht ad hoc ist, auch wenn sie mehr Satztypen als nötig ansetzt. Da es sich - wenn die These richtig ist - bei den Satztypen um tieferliegende Eigenschaften als die besprochenen oberflächenstrukturellen ( \pm Vorfeld mit $\pm w-P h r a s e$, Verbmodus und Tonmuster) handelt, brauchen wir eine Theorie, die mit mehreren syntaktischen Ebenen rechnet und uns damit eine tiefer liegende Ebene bereitstellt, auf der die Satztypen festgelegt werden können. Eine solche Theorie ist die Rektions- und BindungsTheorie (GB-Theorie, Chomsky 1981; 1986). Damit soll nicht behauptet werden, daß es nicht auch andere Theorien gäbe, die Ähnliches leisten könnten. Meines Wissens gibt es aber keine andere Theorie, die gerade in dem hier aktuellen Bereich so detailliert ausgearbeitet ist.

Worin liegen nun dann also die Vorteile der GB? Die Antwort lautet: Mit der Unterscheidung einerseits zwischen mehreren syntaktischen Repräsentationsebenen, vor allem zwischen D- und S-Struktur, andererseits zwischen funktionalen und lexikalischen Köpfen (siehe hierzu Fukui (1986), Abney (1987), Zimmermann (1990)) gibt sie uns ein Instrumen- 
tarium an die Hand, das für die Definition und Erklärung der Satztypen besonders gut geeignet ist.

Auf der D-Struktur-Ebene erlaubt sie uns, eine für alle Sätze einheitliche Struktur anzusetzen und die oberflächenstrukturellen Eigenschaften aus der unterschiedlichen Besetzung der Köpfe und Spec-Positionen abzuleiten. Damit können wir einerseits der intuitiv empfundenen Verwandtschaft zwischen bestimmten "Satztypen" Rechnung tragen, andererseits den oben bemerkten oberflächenstrukturellen Zusammenfall zwischen Satztypen als strukturelle Homonymie erklären. Die D-Struktur des Satzes sieht folgendermaßen aus:

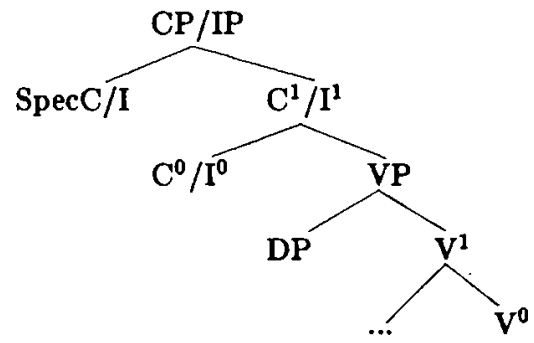

Der Einfachheit halber wird von einer einheitlichen CP/IP-Struktur mit $\mathrm{C}^{0} / \mathrm{I}^{0}$ links von VP ausgegangen und an dieser Stelle keine Diskussion darüber geführt, ob jeder déutsche Satz tatsächlich einen CP-Knoten aufweist (zu dieser Frage, siehe u.a. Reis (1985), Stechow/Sternefeld (1988), Reis/Rosengren (1991c) und Brandt/Reis/Rosengren/Zimmermann (1992)). Andeutungsweise soll nur darauf hingewiesen werden, daf es sich um die alte Diskussion handelt, inwiefern Verb-erst/Verb-zweitSätze I-Projektionen und Verb-letzt-Sätze C-Projektionen sind.

$\mathrm{C}$ und I sind funktionale Köpfe. Die Spec-Position ist - wie allgemein angenommen - optional. Wenn die SpecC-Position vorliegt, können XPPhrasen durch w-Bewegung dorthin bewegt werden. Die Bewegung des Verbs nach $C^{0} / I^{0}$ ergibt Verb-erst- und Verb-zweit-Sätze. Wenn das Verb in situ bleibt, erhalten wir Verb-letzt-Sätze. Die sich so ergebenden Strukturtypen entsprechen jedoch nicht den gesuchten Satztypen. Die Verb-Bewegung korreliert mit der Einbettbarkeit des Satzes - was eine ganz andere Unterteilung der Sätze ergibt als die gesuchte - und die \pm Existenz der SpecC-Position scheint eher eine Einteilung von Subtypen innerhalb der grundlegenden Satztypen Deklarativsatz und Interrogativsatz zu ergeben. 
Um Satztypen zu definieren, brauchen wir über die CP/IP-Struktur hinaus also Merkmale, die uns die Haupttypen liefern. Hier sind die funktionalen Köpfe von Interesse. Es soll angenommen werden, daB die Satztypmerkmale im obersten funktionalen Kopf sitzen.

Ausgehend von diesen Annahmen, gibt es keinen Grund, Verb-letztSätze aus einer Satztypologie auszuschließen, da sie prinzipiell dieselbe D-Struktur aufweisen wie die Verb-erst- und Verb-zweit-Sätze. Es soll deshalb angenommen werden, daB sie sich hinsichtlich des Satztyps nicht von dem entsprechenden Verb-erst- und Verb-zweit-Satz unterscheiden. Dies gilt auch für Adverbialsätze, die als PPs zu betrachten sind, wobei die Präposition eine CP selegiert (Steube 1987; 1990a). Im folgenden soll jedoch nicht weiter auf diesen Typ eingegangen werden.

Die Frage stellt sich nun, um welche Art von Merkmalen es sich bei der Auszeichnung der Satztypen handelt. Hier lohnt es sich, den w-IS zu betrachten. Wenn sich zeigen läßt, daß man für den w-IS ein unabhängig gerechtfertigtes Merkmal braucht (siehe hierzu Reis/Rosengren (1991a)), das sich auch als Satztypmerkmal eignen würde, wäre dies nicht nur ein gutes Argument, Satztypmerkmale anzusetzen, sondern auch ein Grund, bei einer Satztypologie von genau diesem Merkmal auszugehen.

Für den w-IS ist konstitutiv, daB er mindestens eine interrogative wPhrase enthält, die Skopus über die dem w-IS entsprechende Proposition hat. $\mathrm{Zu}$ den Definitionsmerkmalen des w-IS gehört also nicht nur die interrogative $\mathrm{w}$-Phrase, die sich strukturell und semantisch von den indefiniten w-Phrasen unterscheidet, sondern auch die Markierung der Skopusdomäne. Im Standardfall bedeutet dies, daß die w-Phrase Skopus über den Gesamtsatz hat, den sie einleitet. Wenn dies immer der Fall wäre, brauchten wir zumindest fürs Deutsche kein weiteres Merkmal, um die Skopusdomäne anzuzeigen und man könnte alle Sätze, die durch eine interrogative $w$-Phrase eingeleitet sind, als $w$-Interrogativsätze verbuchen.

So einfach ist es aber nicht: Im Deutschen gibt es Fälle, wo die w-Phrase nicht in Initialstellung steht und trotzdem Skopus über den Gesamtsatz hat, der dann der eigentliche Interrogativsatz ist, und umgekehrt Fälle, wo sie in Initialstellung steht und trotzdem keinen Skopus über den Gesamtsatz hat, den sie einleitet. Der einfachste Fall ist hier die multiple Frage:

(18) Wem hat Peter was wohin getragen?

In diesem Satz haben alle w-Phrasen dieselbe Skopusdomäne wie die satzeinleitende. Hier könnte man immer noch sagen, daß die erste w- 
Phrase die Skopusdomäne auch aller anderen Phrasen markiert.

Ein schwierigerer Fall ist die was-Konstruktion:

(19) Was meinte er, wen wir anrufen sollten?

Offensichtlich hat was hier keine andere Funktion als den Anfang der Skopusdomäne der w-Phrase in dem eingebetteten Satz zu kennzeichnen. Die Frage stellt sich, welchen Status dieses was hat. Ohne auf dieses schwierige Problem nāher einzugehen, kōnnen wir immerhin feststellen, daß was keine gewöhnliche Interrogativphrase des Matrixsatzes ist, wie das folgende Beispiel zeigt:

(20) *Wer meinte was, wen wir anrufen sollten?

Wenn was eine ganz gewöhnliche was-Phrase wäre, müßte sie in einer multiplen w-Frage in situ stehen können. Das kann sie aber nicht. Ein weiteres Indiz für die Sonderstellung von was ist die Position der relevanten w-Phrase in (19). Sie steht zwar in Initialstellung, aber in der Anfangsposition des eingebetteten Satzes, der jedoch ihre Skopusdomäne weder ist noch sein kann, da er kein Interrogativsatz ist, wie der folgende Satz zeigt:

(21) *Er meinte, wen wir anrufen sollten.

Verben wie meinen sind nicht subkategorisiert für Interrogativsätze. Die $w$-Phrase in (19) bleibt mit anderen Worten in einer $\mathrm{Zwischenlandepo-}$ sition (SpecC/I) stehen.

Als Vergleich kann der folgende Satz dienen:

(22) Wem sagte er, wen wir anrufen sollten?

In diesem Satz hat die erste w-Phrase Skopus über den Gesamtsatz und die zweite Skopus über den eingebetteten Satz. Die Phrasen haben also einen Skopus, der mit ihrer Position in Übereinstimmung steht.

Während die w-Phrase in (19) in einer Zwischenlandeposition stehenbleibt und trotzdem Skopus über den Gesamtsatz hat, hat die w-Phrase in (23) trotz ihrer Position am Satzanfang nur Skopus über den eingebetteten Satz:

(23) Wieviel schätz mal, daß das Kleid gekostet hat!

In (23) liegt eindeutig ein Imperativsatz vor. ${ }^{2}$ Die interrogative w-Phrase steht also in einer Position, die kein + w enthalten kann, und sie hat Skopus über einen Satz, in dem sie nicht steht. Dieser Skopus muB irgend-

2 Imperativsätze dieses Typs treten vor allem in süddeutschen Dialekten auf. 
wie ausgezeichnet werden. Es ist sinnvoll, anzunehmen, daB dies durch $+w$ in der Spec-Position des eingebetteten Satzes geschieht (siehe hierzu Reis/Rosengren (1991a, c)).

Man kann also durch die Position der w-Phrase die Skopusdomäne nicht definieren, nicht einmal dann, wenn man davon ausgeht, daf die Phrase sich von anderen w-Phrasen strukturell und semantisch unterscheidet.

Ein Ausweg aus diesem Dilemma könnte eventuell die Logische Form (LF) sein. Aber LF kann w-Phrasen nicht vor bzw. zurück in die Skopusposition bewegen, wenn LF nicht weiB, wohin bewegt werden soll. Natürlich könnte man LF mit einer deskriptiven Liste über alle Satzstrukturmöglichkeiten versehen und dadurch richtige Skopuszuweisungen erzwingen. Eine solche Liste wäre aber sicher nicht beschreibungsadäquat, sondern einfach ad hoc.

Viel einfacher ist es deshalb, mit der Standardtheorie anzunehmen, daß schon in der Syntax ein +w-Merkmal eindeutig anzeigt, von wo aus es sich um einen Interrogativsatz handelt, oder anders ausgedrückt, was die Skopusdomäne der interrogativen w-Phrase(n) ist. Dieses +w steht in der Spec-Position (siehe Brandt/Rosengren/Zimmermann (1989) und Reis/Rosengren (1991a)) des betreffenden Satzes. Mit dem Merkmal +w in der Anfangsposition der Skopusdomäne kann man dann erklären, daß eine Operatorphrase wie die interrogative w-Phrase, die selbst ein $+w$ aufweist, in diese Position geht. Die Existenz eines solchen Merkmals in der Spec-Position des Matrixsatzes erklärt einerseits die Funktion von was in (19); seine Nicht-Existenz in der entsprechenden SpecC-Position in (23) erklärt andererseits, daB die w-Phrase nicht Skopus über den Gesamtsatz hat.

Wir brauchen also $+w$, um den Skopusbereich der w-Phrase festzulegen. Ein Satz mit einem $+w$ ist immer zugleich auch ein Interrogativsatz. Wir können deshalb das Merkmal auch als Satztypmerkmal betrachten, das zusammen mit der + w-Phrase den w-IS als einen Interrogativsatztyp definiert. Jeder Satz mit $+w$ in der satzinitialen Position ist also per definitionem ein Interrogativsatz.

Wenn wir nun mit $+w$ den w-IS definieren, stellt sich natürlich die Frage, ob es entsprechende Gründe gibt, auch für den E-IS ein +w anzusetzen, das dann den gewünschten Zusammenhang zwischen den beiden Interrogativsätzen abgäbe. In Brandt/Rosengren/Zimmermann (1989) und Reis/Rosengren (1991a) wurde dies angenommen. Ein erster Grund für eine solche Annahme sind die Subkategorisierungsverhältnisse: 
(24) Ich weiß nicht, ob er ihn gesehen hat.

(25) Ich weiß nicht, wen er gesehen hat.

Daß dieselben Matrixprädikate im Regelfall sowohl w-IS als auch E-IS einbetten, erklärt sich am leichtesten dadurch, daß sie für $+w$ subkategorisiert sind. Entsprechend sind sie „Inseln" für lange Bewegung:

(26) *Wohin weiß Peter, mit wem Hans gereist ist.

(27) *Wohin weiß Peter, ob Hans gereist ist.

Die +w-Phrase kann in beiden Fällen nicht aus dem eingebetteten Interrogativsatz herausbewegt werden, wie dies beim entsprechenden eingebetteten daß-Satz möglich ist.

Ein weiterer Grund, auch für E-IS +w anzunehmen, sind die Verberst-Deklarativsätze (vgl. (2)). Sicher handelt es sich beim Verb-erstDeklarativsatz nicht um eine Vorfeldellipse, wie die folgenden Beispiele zeigen:

(28) Plötzlich kam da ein Mann zur Tür herein.

(29) Kam da plötzlich ein Mann zur Tür herein.

In (29) fehlt kein Satzglied. Der Satz könnnte oberflächenstrukturell ebensogut ein E-IS sein oder anders ausgedrückt: der E-IS und der Verb-erstDeklarativsatz sind oberfächenstrukturell homonym. Die strukturelle Homonymie kann nun dadurch expliziert werden, daß wir die beiden Sātze auf je unterschiedliche D-Strukturen zurückführen, wobei der Interrogativsatz das Merkmal +w bekommt. Damit haben wir aber auch die Brücke geschlagen, die die beiden Interrogativsätze verbindet: nicht die Struktur als solche, sondern das +w-Merkmal ist das Verbindungsglied.

Wir haben aber darüber hinaus auch die Tür für eine Merkmalcharakterisierung des Deklarativsatzes geöffnet. Wenn die Interrogativsätze $+w$ haben, scheint die Annahme nahezuliegen, daß der Deklarativsatz -w hat. Der Unterschied zwischen dem E-IS und dem Verb-erst-Deklarativsatz wäre dann ein Unterschied zwischen $+w$ und -w. Der Unterschied zwischen einem Verb-erst- und einem Verb-zweit-Deklarativsatz wäre wiederum die Existenz/Nicht-Existenz einer SpecC/I-Position. Dies zeigt nochmals deutlich, daß die Existenz/Nicht-Existenz dieser Position verglichen mit dem $\pm w$-Merkmal eine untergeordnete Rolle spielt. Sie kommt erst bei der Subklassifizierung von Satztypen unterhalb der Haupttypen zum Tragen.

Die Konstellation von $\pm w$ beim Deklarativ- und Interrogativsatz sieht demnach folgendermaßen aus: 
(30)

(31) V-erst-Dekl.satz:

$\left.l_{C^{1} / I^{1}} C_{-w}^{0} / I^{0}[\ldots]\right]$

(32) V-zweit + V-letzt-w-IS:

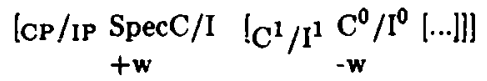

(33) V-erst + V-letzt-E-IS:

$\left.\left.\right|_{C^{1} / I^{1}} C_{+w}^{0} / I^{0}[\ldots]\right)$

Zu diskutieren wäre hier, weshalb der w-IS -w in der Kopfposition hat. Möglich wäre auch $+w$. Welche These hier die beste ist, kann im Rahmen dieses Beitrags nicht diskutiert werden. Es soll jedoch darauf hingewiesen werden, daB -w am besten mit der Tatsache korreliert, daß z.B. im Bairischen im Verb-letzt-Satz in dieser Position nur daß möglich ist:

(34) Sag mir, wen daß du kennst.

Der Unterschied zwischen dem Verb-zweit-/Verb-erst-Satz und dem Verb-letzt-Satz liegt also nicht in der Merkmalcharakterisierung, sondern in der lexikalischen Besetzung der $\mathrm{C}^{0} / \mathrm{I}^{0}$-Position .

Was nun aber mit dem Imperativsatz? Er ist bisher in der GB-Literatur vernachlässigt worden. Offensichtlich hat er im Deutschen ein optionales Vorfeld (siehe (16) oben). Diese Position wird beim Imperativsatz nur im markierten Fall besetzt. Darin unterscheidet er sich vom Verbzweit-Deklarativsatz. Er kommt auch nicht eingebettet bzw. mit Verbletzt vor. Es wäre nun logisch, wenn die Eigenart des Imperativsatzes gegenüber den beiden bisher identifizierten Satztypen einerseits strukturell (z.B. durch das Fehlen eines $C^{0}$-Kopfes, was die Nicht-Einbettbarkeit erklären könnte), andererseits durch ein ganz anderes Merkmal definiert würde, so daß wir der näheren Verwandtschaft zwischen dem Deklarativsatz und dem Interrogativsatz gegenüber dem Imperativsatz Genüge tun und zugleich den Imperativsatz positiv definieren könnten. Der Imperativsatz unterscheidet sich von den beiden anderen Satztypen dadurch, daf er durch einen eigenen Verbmodus gekennzeichnet ist. Der Verbmodus Imperativ hat also hier eine andere Funktion als die Verbmodi Indikativ/Konjunktiv bei den anderen Satztypen oder anders ausgedrückt: der Imperativsatz setzt sich durch die Korrelation zwischen Satztyp und Verbmodus von den beiden anderen Satztypen systematisch ab. Seine Einordnung in ein auf GB-Hintergrund aufgebautes Satztypmodell ist 
eine schwierige Aufgabe, die im Rahmen dieses Beitrags nicht gelöst werden kann. Siehe hierzu jedoch Rosengren (1992c).

Mit dem obigen Instrumentarium können wir die drei traditionell auseinandergehaltenen Satztypen, den Deklarativsatz, die beiden Interrogativsätze und den Imperativsatz, unterscheiden und zugleich ihren Verwandtschaftsgrad explizieren. Da das Merkmalpaar \pm w hierbei eine entscheidende Rolle spielt, sollen noch einige Überlegungen zu seinem Status angefügt werden.

Ausgehend vom w-IS wurde oben gezeigt, daß die Annahme des Merkmals $+w$ Ordnung in die sonst ad hoc erscheinenden Skopusverhältnisse bringt. Das Merkmal +w wurde also vorerst nicht mit Hilfe von satztypologischen Argumenten begründet. Seine Position am Satzanfang und sein Bezug zu der $+w-P h r a s e-b z w$. deren Initialstellung - gibt jedoch Anlaß zu der Überlegung, ob es sich nicht tatsächlich primär um ein Satztypmerkmal handelt, das - außer Satztypmerkmal zu sein - auch für die Skopuszuweisung verantwortlich ist, indem es im w-IS mit einer interrogativen w-Phrase mit demselben Merkmal systematisch korreliert. Das deutlichste Zeichen für einen Zusammenhang zwischen dem Satztypmerkmal $+w$ und der interrogativen $w$-Phrase ist natürlich die obligatorische Initialstellung der w-Phrase im Standardfall. Möglicherweise könnte dies so generalisiert werden, daB die beiden Merkmale $\pm w$ zwei Haupttypen oder Grundtypen von Sätzen definieren, die sich wieder durch die Existenz bzw. Nicht-Existenz einer mit dem Satztypmerkmal korrespondierenden Phrase in Subtypen untergliedern lassen. Die zur Diskussion stehenden Phrasen sind also sozusagen satztyprelevante Operatorphrasen, was ihre Sonderstellung gegenüber anderen Operatorphrasen (siehe hierzu Reis/Rosengren (1991c)) erklären könnte.

2.3. Bisher wurden nur drei grundlegende Satztypen (mit jeweiligen Subtypen) unterschieden. Die nächste Frage, die zu beantworten ist, ist die folgende: Gibt es - wie die traditionelle Grammatik meint - noch weitere Satztypen? Kandidaten sind hier der Echo-w-Satz, der sogenannte Exklamativsatz und der Wunschsatz. Zu diesen Typen ist schon viel geschrieben worden. Hier kann nur kurz angedeutet werden, wie die Antwort im Rahmen des dargestellten Modells aussieht.

Wie Reis (1991a; 1992) zeigt, gibt es keinen eigenständigen Echo-wSatz. „Echo-w-Sätze” sind Deklarativsätze, Interrogativsätze oder Imperativsätze mit einer hauptbetonten $+w-P h r a s e$, die nicht Skopus über den Gesamtsatz hat.

(35) Er hat WEN gesehen?

(36) WEM hat er ein Buch geschenkt? 
(37) Wem hat er WAS geschenkt?

(38) Hat er ihn WO getroffen?

(39) Laß WAS liegen?

Dabei kann die $+w-P h r a s e$ prinzipiell sowohl in situ als auch am Satzanfang stehen. Die einzige Restriktion bezüglich der Position folgt genau aus der Annahme, daB der Echo-w-Satz kein eigener Satztyp ist: Ein selbständiger Satz mit einer hauptbetonten satzinitialen +w-Phrase kann selbstredend nur dann als Echo-w-Satz interpretiert werden, wenn er kein w-IS ist - wenn er also kein +w in SpecC aufweist. Denn +w in der SpecC-Position erzwingt die Bewegung einer +w-Phrase, die Skopus über den Gesamtsatz hat, in die satzinitiale Position, und die Kombination aus $+w$ und satzinitialer Stellung einer $+w-P h r a s e$ ergibt deshalb auch immer einen w-IS. Die Oberflächenstruktur eines Satzes wie (36) ist also strukturell homonym: es handelt sich entweder um einen w-IS mit einer +w-Phrase, die Skopus ùber den Gesamtsatz hat, oder um einen Echo-w-Satz mit -w in der satzinitialen Position, dessen w-Phrase folglich keinen Skopus über den Gesamtsatz hat. Die empirischen Daten bestätigen diese Hypothese (siehe Reis 1991a; 1992)).

Beim Exklamativsatz verhält es sich entsprechend. Immer wieder - zuletzt von Fries (1988) und Rosengren (1992a) - ist auf die Tatsache hingewiesen worden, daB anscheinend jeder Satzstrukturtyp (Verb-erst/Verb-zweit-/Verb-letzt-Satz mit und ohne einleitende w-Phrase) exklamativ verwendet werden kann. Aus Platzgründen wird hier auf Rosengren (1992a) verwiesen, wo ausführlich dafür argumentiert wird, daß exklamative Äußerungen immer entweder Deklarativsätze oder Interrogativsätze sind und daß es sich dabei um ein systematisches Zusammenwirken zwischen Satztyp und weiteren charakteristischen (propositionalen und prosodischen) Eigenschaften der Sätze handelt. Folgende Sätze können/müssen bzw. können nicht (*) exklamativ interpretiert werden:

(40) Die ist vielleicht/aber schön!

(41) Ist die vielleicht/aber schön!

(42) Hab ich aber Hunger!

(43) Bist du vielleicht gewachsen!

(44) $D a \beta$ die so schön ist!

(45) Wie schön die doch ist!

(46) Wie die doch schön ist!

(47) Was ist die doch schōn!

(48) *Was schön die doch ist!

(49) Wen die alles kennt!

(50) *Wen kennt die alles!

(51) Ist die (*aber) nicht schōn! 
(52) Ist die (*aber) nicht gewachsen!

(53) $*$ Hab ich nicht Hunger!

(54) *Bist du nicht gewachsen!

(55) Wen die nicht alles kennt!

(56) Wen kennt die nicht alles!

In (40)-(50) handelt es sich um Deklarativsätze mit und ohne w-Phrase, in (51)-(56) um Interrogativsätze sowohl vom Typ E-IS als auch vom Typ w-IS. Die Interrogativsätze enthalten die Negation nicht und sind nur über eine "rhetorische" Uminterpretation exklamativ interpretierbar. Der Unterschied zwischen dem exklamativen Deklarativsatz und dem E-IS wird auch in der unterschiedlichen Modalpartikelsektion deutlich. Weiter sind bestimmte propositionale Gehalte nur beim Deklarativsatz möglich ((42)-(43)). Es gibt also keinen Grund, einen eigenständigen Exklamativsatztyp anzusetzen. Es handelt sich genau genommen um eine besondere Anwendung von Deklarativsätzen und Interrogativsätzen, also letztendlich um eine pragmatische Kategorie. Die Analyse erklärt nun auch die Zwischenstellung, die dieser Satztyp in den meisten Grammatiken einnimmt: einerseits wird ein exklamativer Satztyp (Ausrufesatz) gewöhnlicherweise angesetzt, andererseits wird auf die nahe Verwandtschaft dieses Satztyps mit dem Deklarativsatz und zuweilen auch mit dem Interrogativsatz hingewiesen.

Auf den Wunschsatz werde ich nicht eingehen können. Wie schon in Brandt/Rosengren/Zimmermann (1989) vorgeschlagen, bin ich der Meinung, daß es sich auch hier nicht um einen eigenständigen Satztyp handelt, sondern um den Deklarativsatz, der seine bestimmte Funktion durch weitere Spezifizierung erhält.

\section{Zum Begriff des Satzmodus}

In Abschnitt 3 sollte gezeigt werden, daB eine modular orientierte Syntaxtheorie wie die GB nicht nur die Unterschiede, sondern auch die Übereinstimmungen zwischen den schon traditionell angesetzten Satztypen konsistent $z \mathfrak{u}$ beschreiben erlaubt. In diesem Abschnitt soll nun der Frage nachgegangen werden, was man auf diesem Hintergrund sinnvollerweise unter Satzmodus verstehen kann.

Die bisher allgemein vorkommende Annahme ist die, daß der Satztyp eine Sprechereinstellung wiedergibt. Ein Versuch, diese Hypothese zu erhärten, findet sich bei Pasch (1990a, b). Sie definiert den Begriff des Satzmodus als ${ }_{n}$ aktuelle ausgedrückte Sprechereinstellung zu einem vom Satz bezeichneten Sachverhalt". Wie Pasch selbst feststellt, zwingt die Hypothese u.a. zu der weiteren Annahme, daß nur selbständige 
Verb-erst- und Verb-zweit-Sätze einen Satzmodus haben. Eingebettete Verb-letzt-Sätze drücken offensichtlich keine Sprechereinstellung aus und können deshalb auch keinen Satzmodus haben. Dies gilt dann konsequenterweise auch für syntaktisch selbständig verwendete Verb-letzt-Sätze. Diese notwendige Konsequenz sollte eigentlich schon genügen, um Zweifel an der Definition des Satzmodus als Sprechereinstellung aufkommen zu lassen. Denn wie kann man erklären, daß die Sprechereinstellung einmal zur grammatisch determinierten Bedeutung des Satzes gehört, ein andermal - beim selbständig verwendeten Verb-letzt-Satz - erst auf der kommunikativen Ebene hinzutritt? In unserem Modell, wo die Satztypen auf der D-Struktur-Ebene definiert werden, kann man natürlich dem Verb-letzt-Satz den Satzmodus nicht absprechen. Wenn der Verb-letztSatz aber auch einen Satzmodus hat, kann der Satzmodus nicht eine Sprechereinstellung sein.

Vor diesem Hintergrund muß untersucht werden, ob man mit einem einstellungsfreien Satzmodusbegriff besser zu Rande kommt. Ein solcher Vorschlag wurde in Brandt/Rosengren/Zimmermann (1989) ausgearbeitet. Der theoretische Rahmen für diesen Vorschlag ist das von Bierwisch (1988) entwickelte kategoriale Semantikmodell. Auf Bierwisch (1988) aufbauend, soll angenommen werden, daß ein Verb im Lexikon nicht nur Argumentstellen für valenzabhängige Argumente bereitstellt, sondern auch eine referentielle Argumentstelle eröffnet, die inı Satz durch die Bedeutung eines funktionalen Kopfes gebunden werden muß. Über diese referentielle Argumentstelle wird also der lexikalische Kopf mit dem funktionalen Kopf kompositionell verbunden und die virtuelle Referenz des propositionalen Gehalts des Satzes garantiert. Zugleich wird die Grundlage für die einzeinen Satztypen geschaffen. Jeder Satz weist also eine Struktur mit einer gebundenen referentiellen Argumentstelle auf. Darüber hinaus enthält er aber weitere Merkmale, die ihn bezüglich des Satztyps spezifizieren. Das bedeutet, daß wir von einer homomorphen Abbildung der Syntax auf die Semantik ausgehen und deshalb auch drei den drei Satztypen entsprechende Satzmodi (mit entsprechenden Submodi) annehmen können: den Deklarativsatzmodus, den Interrogativsatzmodus und den Imperativsatzmodus.

Hier sollen nur die beiden ersteren näher beschrieben werden (zu dem letzteren, siehe Rosengren 1992b): Als syntaktische Kennzeichnung des $\mathrm{C} / \mathrm{I}-\mathrm{Kopfes}$ wurde oben das Merkmalpaar $\pm \mathrm{w}$ angenommen. Entsprechend ist nun der Satzmodus des Deklarativsatzes:

(57) $[\lambda \mathrm{Q}[\exists$ e $[\mathrm{Q}$ e] $]]$ 
d.h. (57) ist die Bedeutung von $\mathrm{C}^{0} \mathrm{I}^{0}$ mit der Kennzeichnung - w, ganz analog zur Artikelbedeutung in Substantivgruppen (siehe hierzu Zimmermann $(1987 ; 1988 ; 1990))$. Der Satzmodus des E-IS ist entsprechend:

(58) $[\lambda$ Q [OFFEN [ᄏ e [Q e]]]]

wobei OFFEN kein Prädikat, sondern ein Operator des Typs S/S (wie die Negation) ist. Der Satzmodus des w-IS ist - dem oben definierten Subtyp entsprechend - das Ergebnis der Amalgamierung der Bedeutung der + w-Phrase, im Falle von z.B. wer $=(59)$,

(59) $[\lambda$ Q [OFFEN x [Person $x]][Q \quad x]]$

mit (57). Der Operator OFFEN in der w-Phrase ist hier vom Typ ((S/S)/S)/N. OFFEN bedeutet in beiden Fällen, daß der Referent nicht identifizierbar ist.

Durch diese Formeln wird nun unserer Intuition - die wohl auch der traditionellen Grammatik zugrundeliegt - hinsichtlich der Unterschiede und Übereinstimmungen zwischen dem Deklarativsatz und den beiden interrogativen Subtypen Rechnung getragen. Der Deklarativsatz ist der Defaulttyp, der genau genommen keine Bedeutung hat, die nicht auch anderen Satztypen zukommt. Er ist sozusagen der unmarkierte Satztyp. Man kann es auch so ausdrücken, daß der Existenzoperator, indem er die Variable 'e' abbindet, die lexikalisch spezifizierte Struktur zu einem Satz macht. Bei den Interrogativsätzen kommt der Operator OFFEN hinzu und gibt ihnen eine eigenständige Bedeutung. Dieser Unterschied zwischen dem Deklarativsatz und den Interrogativsätzen weist auf eine Explikationsmöglichkeit der sonst schwer erklärbaren Funktionsfestigkeit der Interrogativsätze gegenüber den Deklarativsätzen hin. Vielleicht ist auf diesen Unterschied auch die Tatsache zurückzuführen, daß man mit dem $d a \beta$-Satz keine Assertion, mit dem selbständigen $o b$ - und w-Verbletzt-Satz dagegen Fragen vollziehen kann.

Zusammenfassend können wir also feststellen: Das oben entworfene Modell beschreibt die Abbildung der Syntax auf die Semantik und wie Satzmodus und propositionale Bedeutung miteinander kompositional verbunden werden. Das Ergebnis ist eine grammatisch determinierte Satzbedeutung, die sowohl den Satzmodus als auch die propositionale $\mathrm{Be}$ deutung des Satzes umfaßt.

\section{Die Beziehung zwischen Satzmodus und Illokution}

Die vorgeführte Satzmodustheorie ist die denkbar einfachste und generellste. Sie ist einfach, insofern sie ein bestimmtes Satztypınerkmal mit einer bestimmten Bedeutung verbindet und diese Bedeutung als einen 
bestimmten Operator definiert. Sie ist generell, insofern sie alle Sätze auf dieselbe Weise behandelt und weder zwischen den Satztypen unterscheiden muß, indem sie z.B. den Deklarativsatz einstellungsfrei und die anderen mit Einstellungen definiert, noch bestimmte Satzstrukturen ganz von der Semantik ausschließen muß. Ihre Haltbarkeit ist aber sehr davon abhängig, ob es zu erklären gelingt, wie der Satz, ausgestattet durch eine solche Semantik, die prototypische Illokution findet, mit der man ihn normalerweise assoziiert.

Diese Frage ist ausführlich von Rehbock $(1989)^{3}$ behandelt worden. Hier soll nur auf seine Analyse des Deklarativsatzes eingegangen werden, teils weil dieser Satztyp wohl der Defaulttyp ist, teils aber auch, weil er das gröBte Ilokutionspotential aufweist. Nach Rehbock ist

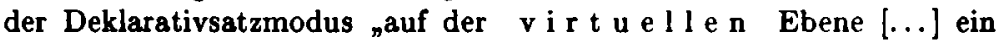
potentielles Refere $\mathrm{nz}$ it te l, mit dem ein Sprecher bei der Aktualisierung des Satzes den (die Proposition instantiierenden) Sachverhalt e auf einen Zustand/ein Ereignis in der 'Welt' beziehen kann. Auf der a k t u e ll e n Ebene der Sprachhandlung tut der Sprecher eben dies als Teil des rhetischen Aktes: Er 'projiziert' e als modal und temporal spezifiziertes Fakt in die derzeitige Diskurswelt und gibt dem Hörer die 'Anweisung', diesen Sachverhalt dort ebenfalls als vom Sprecher identifiziertes Fakt zu lokalisieren". Dies erlaubt noch keine illokutive Interpretation. Es muß vom situativen Kontext ein weiteres Merkmal beigesteuert werden, das uns entscheiden läßt, ob der Sachverhalt unabhängig vom Sprecher existiert oder nicht. Rehbock unterscheidet hier - in Anlehnung an eine von Motsch/Pasch (1987) vorgeschlagene Distinktion - zwischen unterschiedlichen rhetischen Spezifizierungen oder Referenzmodi, nämlich den konstativen und den konstitutiven Âuerungen: die Existenz des von der Proposition denotierten Fakts ist unabhängig vom Sprecher bzw. die Existenz des Fakts ist vom Sprecher oder von der sprachlichen Handlung abhängig.

Im Falle der konstativen Äußerungen gilt dann die Grundvoraussetzung, daß man nicht auf ein vom Sprecher unabhängiges Fakt verweisen kann, ohne die Überzeugung von der Existenz jenes Fakts zum Ausdruck zu bringen, nämlich "daß der ausgedrückte propositionale Gehalt w a h r ist". Mit einer konstativen Äußerung vollzieht der Sprecher deshalb auch eine Assertion:

(60) Peter hat die Fahrkarten gekauft.

3 Siehe jetzt auch Rehbock (1992a). 
Die konstitutiven Äußerungen unterteilen sich wiederum in streng gleichzeitige - performative - Fälle und in nachzeitige - resultative - Fälle (siehe auch Liedtke (1989)). Die ersteren sind identisch mit den explizit performativen Äußerungen:

(61) Ich bitte dich, dies nie wieder zu tun.

(62) Ich behaupte, daß es sich so verhält.

Die letzteren umfassen Beispiele wie:

(63) Dies ist ein Schiff. (z.B. in einem Spielkontext)

(64) Karl übernimmt ab morgen das Kommando. (als Befehl)

(65) Ich werde den Sekt nicht vergessen. (als Versprechen)

Die Beispiele (61)-(65) sind insofern ambig, als sie auch als ganz normale konstative Äußerungen und damit als Feststellungen aufgefaßt werden können. Im Falle der performativen Äußerungen (61)-(62) geschieht dies nur im markierten Fall (d.h. in bestimmten Kontexten). Bei (63)-(65) hängt die Möglichkeit, sie konstitutiv zu interpretieren, sehr von dem propositionalen Gehalt ab. In einem geeigneten Kontext kann (63) also deklarativ, (64) direktiv und (65) kommissiv interpretiert werden.

Die Referenzmodi sind also die Brücke, auf der der Sprecher vom Satzmodus eines Satzes zu seiner illokutiven Interpretation kommt. Von der Perspektive des Referenzmodus aus gesehen, gibt es deshalb auch eine systematische Beziehung zu den grundlegenden Illokutionen. Die Ilokution ist jedoch ein weiterer Begriff, indem noch Einstellungen, Obligationen und Ziele des Sprechers relativ zum Adressaten (die konventionell determinierte Interaktionsseite des Sprechakts) hinzukommen.

Mit dieser Explikation des Zusammenhangs zwischen Satzmodus, Referenzmodus und Ilokution im Bereich des Deklarativsatzes soll also gezeigt werden, dab es keineswegs notwendig ist, einen Einstellungsoperator schon in der Semantik anzusetzen. Die Einstellung ist ein Bestandteil der Ilokution und steht in einer festen Beziehung zu dem Referenzmoduspotential des Satzes. Der Bezugspunkt zwischen Satzmodus und Illokution ist der Referenzmodus, der die virtuelle Referenz des Satzes auf einen Sachverhalt mit der aktuellen Referenz auf einen bestimmten Sachverhalt und damit mit der jeweiligen nlokution verbindet. Das Modell erlaubt uns also, sauber zwischen Grammatik und Pragmatik zu trennen.

Die weiteren Analysen von Rehbock $(1991 ; 1992 b)$ zum Interrogativsatz zeigen, daß sich eine ähnliche Erklärung auch bei diesem Satztyp bewährt. 
Der Imperativsatz ist nicht nur syntaktisch, sondern auch semantisch komplizierter als die beiden anderen Satztypen, weil es sich um einen deontischen Satzmodus handelt. Auch hier braucht man aber meines Erachtens keinen Einstellungsoperator anzunehmen (siehe Rosengren 1992c). Der Sprecher und seine Einstellungen gehören somit generell in die Pragmatik und werden auch erst dort angesteuert.

\section{Ausblick}

Das oben entwickelte Modell ist eine Skizze. Viel Arbeit steht noch aus, ehe die Interaktion zwischen den Modulen expliziert ist. Auf der grammatischen Seite muß das Zusammenwirken von Satztyp und Satzmodus mit dem propositionalen Gehalt genauer untersucht werden. Auf der pragmatischen Seite ist noch zu klären, was eine Illokution eigentlich ist, welche Ilokutionstypen es gibt und wie ein Illokutionssystem in einen größeren pragmatischen Zusammenhang einzuordnen ist. Von besonderem Interesse sind dabei auch Einstellungsausdrücke und Modalpartikeln, die mit den Illokutionstypen auf eine systematische Weise interagieren. Einige dieser Fragen werden in den einzelnen Beiträgen in Rosengren (1992b) eingehender diskutiert.

\section{Literatur}

Abney, Steven (1987): The English Noun Phrase in its Sentential Aspect. Doctoral diss. MIT, Cambridge/MA.

Altmann, Hans (1990): Satzmodus. Erscheint in: Jacobs, Joachim et al. (Hg.)(i.V.): Handbuch der Syntax.

Altmann, Hans/Batliner, Anton/Oppenrieder, Wilhelm (Hg.) (1989): Zur Intonation von Modus und Fokus in Deutschen. Tübingen. (= Linguistische Arbeiten 234).

Bierwisch, Manfred (1979): Wörtliche Bedeutung. Eine pragmatische Gretchenfrage. In: Rosengren, Inger (Hg.): Sprache und Pragmatik. Lund. (= Lunder germanistische Forschungen 48), S. 63-85.

Bierwisch, Manfred (1980): Semantic Structure and Illocutionary Force. In: Searle, J.R./Kiefer, F./Bierwisch, M. (Hg.): Speech Act Theory and Pragmatics. Dordrecht. S. 1-35.

Bierwisch, Manfred (1988): On the Grammar of Local Prepositions. In: Bierwisch, Manfred/Motsch, Wolfgang/Zimmermann, Ilse (Hg.): Syntax, Semantik und Lexikon. Berlin. (= studia grammatica XXIX), S. 1-66. 
Brandt, Margareta/Reis, Marga/Rosengren, Inger/Zimmermann, Ilse (1992): Satztyp, Satzmodus und Illokution. In: Rosengren, Inger (Hg.) (1992b), Band 1, S. 1-89.

Brandt, Margareta/Rosengren, Inger/Zimmermann, Ilse (1989): Satzmodus, Modalität und Performativität. In: Sprache \& Pragmatik, H. 13, S. 1-42. (Auch in: Zeitschrift für Phonetik, Sprachwissenschaft und Kommunikationsforschung, Jg. 1990, H. 43, S. 120-149.)

Chomsky, Noam (1981): Lectures on Government and Binding. Dordrecht.

Chomsky, Noam (1986): Barriers. Cambridge/MA.

Duden Band 4: Grammatik der deutschen Gegenwartssprache (1984). 4. Aufl. hrsg. von G. Drosdowski. Mannheim/Wien/Zürich.

Fries, Norbert (1988): Ist Pragmatik schwer! - Über sogenannte „Exklamativsätze” im Deutschen. In: Sprache \& Pragmatik, H. 3, S. 1-18. (Auch in: Deutsche Sprache 16 (1988), S. 193-205.)

Fries, Norbert (1992): Interjektionen, Interjektionsphrasen und Satzmodus. In: Rosengren, Inger (Hg.) (1992b), Band 1, S. 307-341.

Fukui, N. (1986): A Theory of Category Projection and its Application. Doctoral diss. MIT, Cambridge/MA.

Grewendorf, Günther/Zaefferer, Dietmar (demnächst): Theorien der Satzmodi. Erscheint in: Wunderlich, Dieter/Stechow, Arnim von (Hg.). Handbuch Semantik.

Grundzüge einer deutschen Grammatik (1981). Von einem Autorenkollektiv unter der Leitung von K. E. Heidolph, W. Flämig und W. Motsch. Berlin.

Helbig, Gerhard/Buscha, Joachim (1991): Deutsche Grammatik. Ein Handbuch für den Ausländerunterricht. Leipzig/Berlin/München.

Liedtke, Frank (1989): Performativität, Sprechhandlung, Wahrheit. In: Sprache \& Pragmatik, H. 12, S. 43-67. (Auch in: Zeitschrift für Phonetik, Sprachwissenschaft und Kommunikationsforschung, 1990, H. 4)

Meibauer, Jörg (Hg.) (1987): Satzmodus zwischen Grammatik und Pragmatik. Tübingen. (= Linguistische Arbeiten 180).

Motsch, Wolfgang/Pasch, Renate (1987): Illokutive Handlungen. In: Motsch, Wolfgang (Hg.): Satz, Text, sprachliche Handlung. Berlin. (= studia grammatica XXV), S. 1-79. 
Motsch, Wolfgang/Reis, Marga/Rosengren, Inger (1989): Zum Verhältnis von Satz und Text. In: Sprache \& Pragmatik, H. 11, S. 1-36. (Auch in: Deutsche Sprache 18 (1990), S. 97-125.)

Pasch, Renate (1989): Überlegungen zum Begriff des „Satzmodus". In: Studien zum Satzmodus II. (= Linguistische Studien. Reihe A. Arbeitsbericht 193), S. $1-88$.

Pasch, Renate (1990a): 'Satzmodus' - Versuch einer Begriffsbestimmung. In: Zeitschrift für Phonetik, Sprachwissenschaft und Kommunikationsforschung, H. 43, S. 92-110.

Pasch, Renate (1990b): Satzmodus als Grundlage illokutiver Funktionen: Kritische Fragen zu Brandt/Rosengren/Zimmermann: Satzmodus, Modalität und Performativität. In: Zeitschrift für Phonetik, Sprachwissenschaft und Kommunikationsforschung, H. 43, S. 810-822.

Pasch, Renate (1991): Überlegungen zur Syntax und zur semantischen Interpretation von w-Interrogativsätzen. In: Deutsche Sprache 19, S. 193-212.

Rehbock, Helmut (1989): Deklarativsatzmodus und pragmatische Interpretation. In: Sprache \& Pragmatik, H. 15, S. 1-69.

Rehbock, Helmut (1991): Fragen stellen - Zur Interpretation des Interrogativsatzmodus. In: Reis/Rosengren (Hg.) (1991b), S. 13-47.

Rehbock, Helmut (1992a): Deklarativsatzmodus, rhetische Modi und Illokutionen. In: Rosengren, Inger (Hg.) (1992b), Band 1, S. 91-171.

Rehbock, Helmut (1992b): Fragen stellen - Zur Interpretation des Interrogativsatzmodus. In: Rosengren, Inger (Hg.) (1992b), Band 1, S. 173-211.

Reis, Marga (1985): Satzeinleitende Strukturen im Deutschen. Über COMP, Haupt- und Nebensätze, w-Bewegung und die Doppelkopfanalyse. In: Abraham, Werner (Hg.), Erklärende Syntax des Deutschen. Tübingen. S. 271-311.

Reis, Marga (1991a): Echo-w-Sätze und Echo-w-Fragen. In: Reis, Marga/Rosengren, Inger (Hg.) (1991b), S. 49-76.

Reis, Marga (1991b): Was konstituiert w-Interrogativsätze? Gegen Paschs Überlegungen zur Syntax und Semantik interrogativer w-Konstruktionen. In: Deutsche Sprache 19, S. 213-238.

Reis, Marga (1992): Zur Grammatik und Pragmatik von Echo-w Fragen. In: Rosengren, Inger (Hg.) (1992b), Band 1, S. 213-262. 
Reis, Marga/Rosengren, Inger (1991a): Einleitung. In: Reis, Marga/Rosengren, Inger (Hg.) (1991b), S. 1-11.

Reis, Marga/Rosengren, Inger (Hg.) (1991b): Fragesätze und Fragen. Tübingen. (= Linguistische Arbeiten 257)

Reis, Marga/Rosengren, Inger (1991c): What do wh-Imperatives Tell Us About wh-Movement? Erscheint in: NLLT.

Rosengren, Inger (1988a): Die Beziehung a wischen Satztyp und Illokutionstyp aus einer modularen Sicht. In: Sprache und Pragmatik, H. 6, S. 1-30. (Auch in: Studien zum Satzmodus. II., S. 113-138).

Rosengren, Inger (1992a): Zur Grammatik und Pragmatik der Exklamation. In: Rosengren, Inger (Hg.) (1992b), Band 1, S. 263-305.

Rosengren, Inger (1992b) (Hg.): Satz und Illokution. Band 1 und 2. Tübingen. (= Linguistische Arbeiten S. 278/279).

Rosengren, Inger (1992c): Zur Syntax und Semantik des Imperativsatzes. Erscheint in: Rosengren, Inger ( $\mathrm{Hg}$.$) , Band 2, Satz und Illokution.$

Stechow, Arnim von/Sternefeld, Wolfgang (1988): Bausteine syntaktischen Wissens. Opladen.

Steube, Anita (1987): Grammatical Relations Between Prepositions, Conjunctions and the Complementizer "da $B^{n}$ in a REST-Grammar of German. In: Linguistische Arbeitsberichte 61. Leipzig. S. 54-74. (Erscheint auch in: Proceedings of the XIVth International Congress of Linguists, Berlin 10.-15.8.1987. Berlin).

Steube, Anita (1990a): Die syntagmatische Funktion von "da”, d- und wWörtern. In: Steube, Anita (Hg.) (1990b), S. 200-229.

Steube, Anita (Hg.) (1990b): Syntaktische Repräsentationen mit leeren Kategorien oder Proformen und ihre semantischen Interpretationen. Berlin. (= Linguistische Studien. Reihe A. Arbeitsberichte, 206).

Studien zum Satzmodus. I (1988), II (1988), III (1989). (= Linguistische Studien. Reihe A. Arbeitsberichte 177, 185, 193)

Zimmermann, Ilse (1987): Syntactic Categorization. Erscheint in: Proceedings of the XIVth International Congress of Linguists, Berlin 10.-15.8.1987. Berlin. 
Zimmermann, llse (1988): Wohin mit den Affixen? In: Motsch, Wolfgang (Hg.): Wortstruktur und Satzstruktur. Berlin (= Linguistische Studien, Reibe A, Arbeitsberichte, 194), S. 157-188.

Zimmermann, Ilse (1990): Zur Legitimierung leerer Köpfe. In: Steube, Anita (Hg.) (1990b). S. 75-90.

Zimmermann, Ilse (1991): Die subordinierende Konjunktion wie. In: Reis, Marga/Rosengren, Inger (Hg.) (1991b), S. 113-122. 